\title{
Importância do açaí na renda mensal da comunidade quilombola Murumuru em Santarém, Pará
}

O açaí se destaca como um dos Produtos Florestais Não Madeireiros (PFNMs) mais importantes na região amazônica, por possuir um grande potencial agronômico, tecnológico, nutricional e econômico. Com isso, o objetivo deste trabalho foi avaliar a importância da comercialização do açaí na renda mensal dos moradores da comunidade quilombola Murumuru em Santarém, Pará. Foram realizadas 16 entrevistas com os atores envolvidos na cadeia produtiva do açaí na comunidade Murumuru. Os entrevistados foram selecionados por meio da técnica 'bola de neve'. Como a coleta dos dados foi realizada em 2012, os valores em R\$় (reais) informados pelos entrevistados foram corrigidos e convertidos para dólares americanos (US\$). A atividade de açaí contribui na renda dos quilombolas com US\$ 294,94, contra US\$292,89 dos comunitários que recebem salários; US\$ 124,08 dos comunitários que trabalham com pesca; US\$ 69,15 dos que recebem bolsa do governo; e US\$29,15 dos que se encaixam no grupo 'outros', que engloba tanto quem trabalha com artesanato quanto com serviços de diarista; além de US\$ 58,21 , US\$ 19,06 e US\$33,70 para os que trabalham com taberna, pensão e pecuária, respectivamente. A atividade de açaí gera maior renda familiar mensal em relação às demais atividades remuneradas. Os quilombolas na comunidade Murumuru que comercializam os frutos de açaí in natura possuem maior renda com esta atividade do que aqueles que comercializam a polpa de açaí.

Palavras-chave: Extrativismo; Produto Florestal Não Madeireiro; Comercialização.

\section{Importance of açai in the monthly income of the Murumuru quilombola community in Santarém, Pará}

\begin{abstract}
Açaí stands out as one of the most important Non-Timber Forest Products (NTFPs) in the Amazon region, as it has great agronomic, technological, nutritional and economic potential. Thus, the objective of this work was to evaluate the importance of açai commercialization in the monthly income of the residents of the Murumuru quilombola community in Santarém, Pará. 16 interviews were conducted with the actors involved in the açai production chain in the Murumuru community. The interviewees were selected using the 'snowball' technique. As data collection was carried out in 2012, the amounts in $R \$$ (reais) reported by the interviewees were corrected and converted to US dollars (US \$). Açaí activity contributes to the income of quilombolas with US \$294.94, against US \$292.89 for community members who receive salaries; US $\$ 124.08$ from community workers working with fishing; US $\$ 69.15$ of those receiving government grants; and US $\$$ 29.15 from those who fit in the 'others' group, which includes both those who work with handicrafts and the services of a day laborer; in addition to US $\$ 58.21$, US \$ 19.06 and US \$33.70 for those who work in tavern, pension and livestock, respectively. Açaí activity generates higher monthly family income compared to other paid activities. Quilombolas in the Murumuru community who sell fresh açaí fruits have a higher income from this activity than those who sell açaí pulp.
\end{abstract}

Keywords: Extractivism; Non-Timber Forest Product; Commercialization.

Topic: Ciências Florestais

Reviewed anonymously in the process of blind peer
Received: 03/02/2020

Approved: 08/03/2020
Lucas Cunha Ximenes (iD

Universidade Federal do Oeste do Pará, Brasil http://lattes.cnpq.br/0879648522574450

http://orcid.org/0000-0002-3568-4401

lucasximenesflorestal@gmail.com

João Ricardo Vasconcellos Gama (ic)

Universidade Federal do Oeste do Pará, Brasil

http://lattes.cnpq.br/9058536716453750

http://orcid.org/0000-0002-3629-3437

jrvgama@gmail.com

Talita Godinho Bezerra (iD

Universidade Federal Rural da Amazônia, Brasil

http://lattes.cnpq.br/4945814991752068

http://orcid.org/0000-0001-9968-7874

talita.gbezerra@gmail.com
Girlene da Silva Cruz

Universidade do Estado de Santa Catarina, Brasil

http://lattes.cnpq.br/7126687497486027

http://orcid.org/0000-0001-8023-3338

girlene.lenecruz@gmail.com
Referencing this:

XIMENES, L. C.; GAMA, J. R. V.; BEZERRA, T. G.; CRUZ, G. S..

Importância do açaí na renda mensal da comunidade quilombola Murumuru em Santarém, Pará. Revista Ibero Americana de Ciências Ambientais, v.11, n.2, p.36-42, 2020. DOI:

http://doi.org/10.6008/CBPC2179-6858.2020.002.0004 


\section{INTRODUÇÃO}

Comunidades quilombolas são consideradas populações tradicionais formadas por descendentes de escravos (DIEGUES et al., 2004). Em ecossistemas florestais, os quilombolas se tornaram extrativistas, sendo que as formas de apropriação dos recursos naturais foram, na maioria das vezes, resultado da interação com o ambiente em que se encontravam na medida em que as famílias se refugiavam em áreas de florestas (CARRIL, 2006).

Dentro desse contexto, encaixa-se a extração vegetal de Produtos Florestais Não Madeireiros (PFNMs), que, além de preservar os modos de vida de comunidades tradicionais, é considerada como uma das formas mais sustentáveis de conservação das florestas (REGO, 1999; LESCURE, 2000; ROCHA, 2004; NYGREN et al., 2006), cujos sistemas de produção e uso de recursos naturais são de baixo impacto ambiental, não alterando a dinâmica natural do ecossistema (DIAS, 2001).

O açaí destaca-se na região amazônica como um desses produtos, por possuir um grande potencial agronômico, tecnológico, nutricional e econômico (YUYAMA et al., 2011), além de ter sido o PFNM com maior valor de produção na extração vegetal em 2016 no Brasil, com R\$ 539,8 milhões (IBGE, 2017). O uso do açaí na região amazônica passou de uma situação de alimentação cotidiana das famílias rurais para um hábito alimentar urbano, com expansão nacional e internacional, destacando-se países como Estados Unidos e Austrália (BRONDÍZIO, 2005).

Apesar do alto valor econômico dos produtos e subprodutos do açaí (Euterpe oleracea Mart. Arecaceae) no final da cadeia produtiva, é necessário que sejam realizadas pesquisas evidenciando a remuneração proveniente da comercialização do açaí nas comunidades extrativistas face à outras fontes de renda que as famílias possuem, de modo a incentivar sua produção. Diante disso, o objetivo deste trabalho foi avaliar a importância da comercialização do açaí na renda mensal dos moradores da comunidade quilombola Murumuru em Santarém, Pará.

\section{MATERIAIS E MÉTODOS}

\section{Área de estudo}

O estudo foi realizado na comunidade quilombola Murumuru, localizada no planalto santareno (terra firme) às proximidades da rodovia PA - 370 (Figura 1), distante $30 \mathrm{~km}$ da cidade de Santarém. O clima da região, é do tipo quente e úmido, com temperatura média de $25,5^{\circ} \mathrm{C}$ e precipitação pluviométrica média oscilando em torno de $2000 \mathrm{~mm}$ com distribuição irregular e o período mais chuvoso ocorre entre os meses de dezembro a junho, concentrando mais de $70 \%$ da precipitação anual. Os solos que mais predominam são os Latossolos Amarelos Distróficos e os Argissolos Amarelos Distróficos (EMBRAPA, 2001). Em 2012, época de realização do estudo, moravam 103 famílias na comunidade (SILVA et al., 2011), sendo que destas, 16 tinham participação efetiva na extração e comercialização de açaí (frutos in natura e/ou polpa de açaí). 


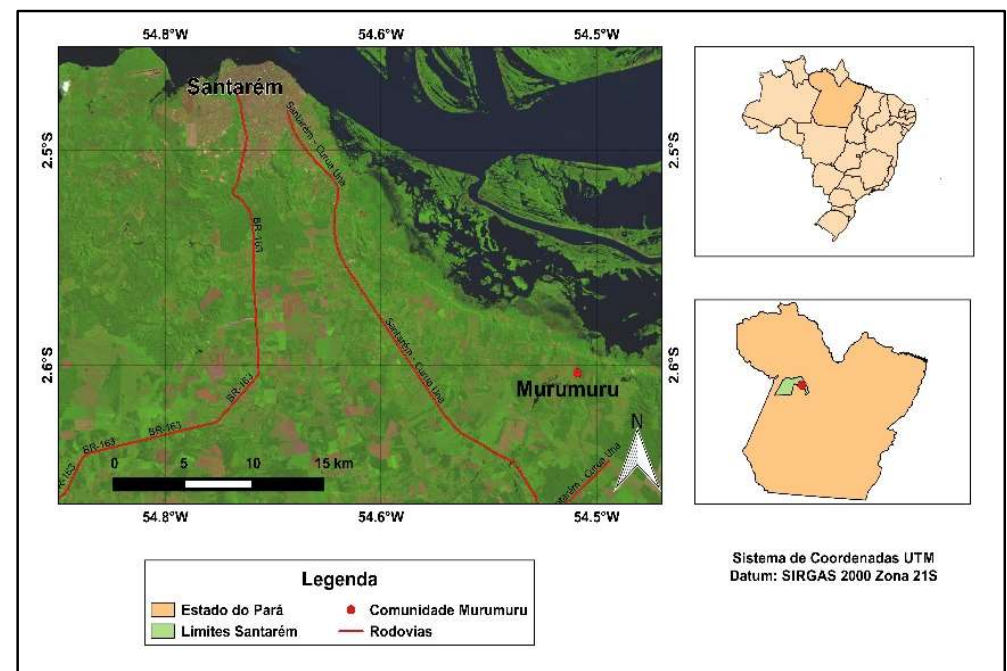

Figura 1: Localização da comunidade quilombola Murumuru em Santarém, Pará.

O acesso à comunidade se dá tanto por via fluvial como terrestre, neste último caso pela rodovia Santarém/Curuá-Una, possuindo ramais de terra que interligam as comunidades entre si. Na estação chuvosa na Amazônia, esse acesso é dificultado, uma vez que há alagamentos de trechos dos ramais, além das condições ruins de trafegabilidade, devido à presença de buracos e deslocamentos de terra (TAKANASHI, 2014).

\section{Coleta e análise dos dados}

Foram realizadas 16 entrevistas com os atores envolvidos na cadeia produtiva do açaí (compreendendo coletores e comerciantes de frutos, atravessadores, processadores e comerciantes da polpa de açaí). Os entrevistados foram selecionados por meio da técnica snowball sampling (BIERNACKI et al., 1981) ou 'bola de neve' como é conhecida no Brasil. Esta técnica é uma forma de amostra não probabilística utilizada em pesquisas sociais, no qual os participantes iniciais de um estudo indicam novos participantes, que por sua vez indicam outros e assim sucessivamente, até que seja alcançado o objetivo proposto (o 'ponto de saturação'). O 'ponto de saturação' é atingido quando os novos entrevistados passam a repetir os conteúdos já obtidos em entrevistas anteriores, sem acrescentar novas informações relevantes à pesquisa (WHA, 1994).

A organização, consistência, análise e processamento dos dados teve caráter explicativo e descritivo, com abordagem quali-quantitativa e realizada com o auxílio de planilhas eletrônicas. Como a coleta dos dados foi realizada entre 15 de junho de 2012 e 17 de junho de 2012, os valores em R\$ (reais) informados pelos entrevistados foram corrigidos e convertidos para dólares americanos (US\$) dessa época. A conversão se deu baseada nas cotações e boletins do Banco Central do Brasil, no qual US\$ 1 foi convertido para R\$ 2,04.

\section{RESULTADOS}

Apesar de todos os entrevistados estarem diretamente envolvidos na cadeia produtiva de açaí na comunidade e do açaí ter grande importância na renda mensal dos comunitários, a atividade de pesca foi considerada a atividade econômica principal com o maior número de envolvidos (42,9\%), seguida do açaí 
(38,1\%), agricultura e pecuária $(9,5 \%)$ cada uma.

Entretanto, o que se percebe é que apesar da atividade de comercialização do açaí não abranger o maior número de comunitários, é a que gera maior renda familiar mensal em relação às demais com US\$ 294,94, contra US\$292,89 dos comunitários que recebem salários; US\$124,08 dos comunitários que trabalham com pesca; US\$ 69,15 dos que recebem bolsa do governo; e US\$ 29,15 dos que se encaixam no grupo 'outros', que engloba tanto quem trabalha com artesanato quanto com serviços de diarista; além de US\$ 58,21, US\$19,06 e US\$ 33,70 para os que trabalham com taberna, pensão e pecuária, respectivamente (Figura 2). Entende-se que os atores englobados no grupo 'taberna', são comerciantes que vendem bebidas alcoólicas e produtos alimentícios em estabelecimentos privados na comunidade.

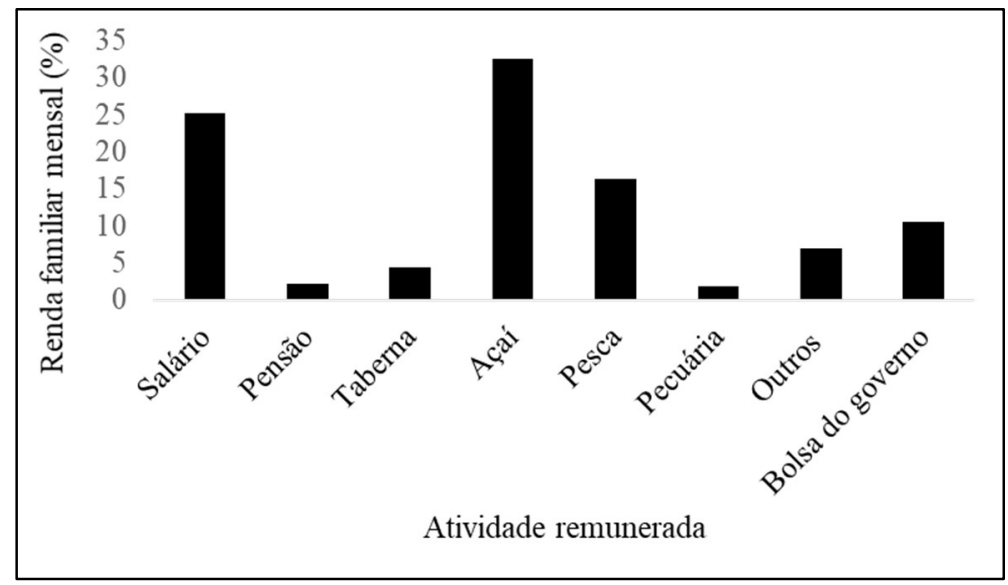

Figura 2: Contribuição percentual das atividades econômicas para composição da renda familiar, comunidade Murumuru, Santarém - Pará.

Como as bolsas do governo tiveram uma participação significativa na renda mensal dos comunitários (81,25\% recebem tal benefício), observou-se que o programa bolsa família apresentou o maior número de beneficiários (93\%), seguido da bolsa escola (7\%), mostrando a importância dos programas do governo federal de transferência direta de renda na vida dos comunitários.

Verificou-se que os atores envolvidos na cadeia produtiva de açaí em Murumuru estão divididos em quatro grupos, aqui denominados de: coletor e comerciante dos frutos in natura (coletam e comercializam somente os frutos), atravessador (compram os frutos e os transportam até o centro consumidor), coletor e comerciante dos frutos in natura e da polpa (coletam e comercializam os frutos in natura e transformam alguns frutos em polpa) e coletor e comerciante da polpa (coletam e comercializam somente a polpa do açaí).

Com isso, percebeu-se que em Murumuru o número de comunitários que coletam e comercializam os frutos in natura é maior que todos os outros envolvidos nas demais atividades, seguido dos atravessadores e dos que coletam e comercializam os frutos e a polpa e dos que coletam e comercializam somente a polpa (Figura 3). Além disso, verificou-se também a contribuição do açaí e de todas as outras rendas mensais por cada ator envolvido na cadeia produtiva de açaí na comunidade Murumuru, com açaí e salários contribuindo com a maior parte da renda (figura 4). 


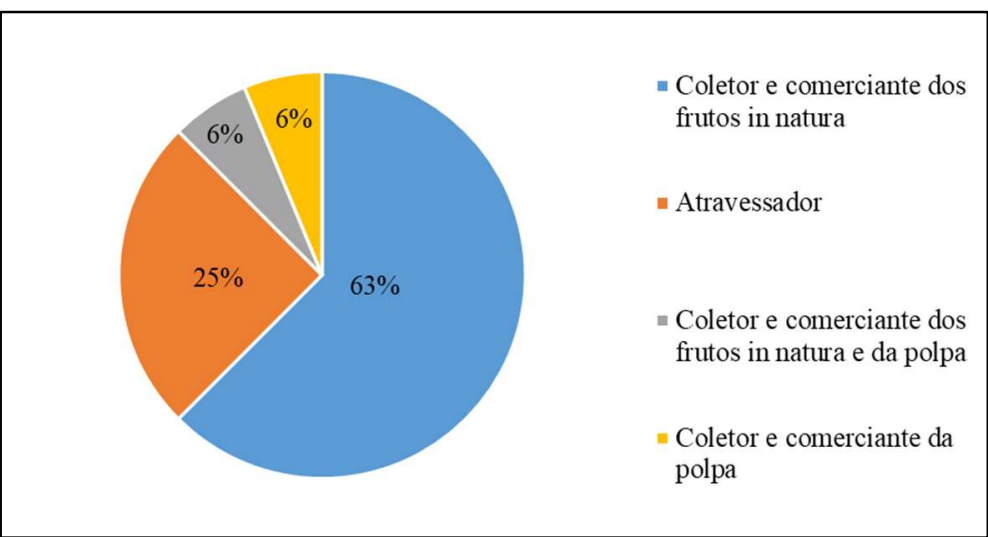

Figura 3: Divisão dos quilombolas nas classes de atuação da cadeia produtiva do açaí, comunidade Murumuru, Santarém - Pará.

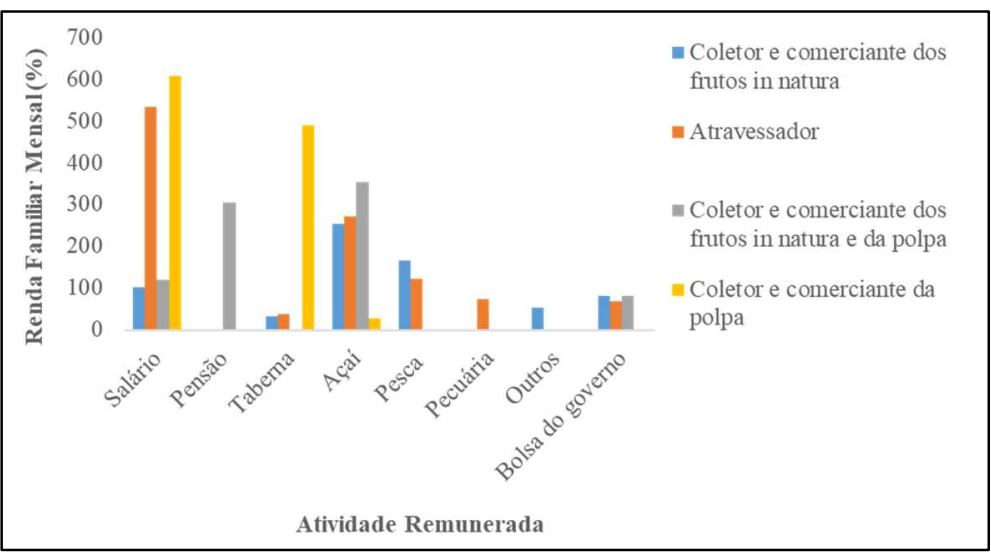

Figura 4: Média da renda familiar mensal das atividades remuneradas por classe de atuação na cadeia produtiva do açaí na comunidade Murumuru, Santarém - Pará.

\section{DISCUSSÃO}

O fato da pesca ser considerada a atividade que envolve o maior número de comunitários corrobora com os resultados encontrados por Silva et al. (2008), no qual a categoria profissional de pescadores é a mais frequente nas comunidades quilombolas de Santarém, Pará. Além disso, Vaz et al. (2017) destacam a pesca na região como a atividade econômica que envolve o maior número de pessoas com a coleta de muitas espécies, como o pacu (Piaractus mesopotamicus), aracu (Leporinus freiderici), tambaqui (Colossoma macropomum) e o tucunaré (Cichla ocellaris), bastante apreciados pela população ribeirinha e que possuem um alto valor comercial.

Entretanto, o açaí é a atividade que mais contribui na renda mensal dos quilombolas de Murumuru, mesmo sendo executada somente em quatro meses do ano (Junho a Outubro - período da safra), diferentemente das demais atividades que contribuem durante todos os meses do ano. Isso pode explicar o fato de que todos aqueles envolvidos na cadeia produtiva do açaí necessitam complementar sua renda mensal com outras atividades, principalmente com empregos, como pode ser visto no elevado índice dos comunitários que recebem salários.

Dois dos maiores contribuintes na renda mensal dos comunitários em Murumuru foram os programas de transferência de renda do governo federal. Dentre estes, o benefício denominado 'bolsa família' também foi o responsável por abranger o maior número de comunitários em outras comunidades 
quilombolas paraenses, como em São José de Icatu (CONCEIÇÃO et al., 2014), e em Abacatal (ARAÚJO et al., 2017), além de apresentar significativa importância em Arapemã, Bom Jardim, Murumuru, Murumurutuba, Saracura e Tiningú (SILVA et al., 2008).

As vantagens desse benefício vão muito além de ser uma simples fonte de renda. Pedroso Júnior et al. (2008), estudando a socioeconomia e a demografia de nove comunidades quilombolas em São Paulo, detectaram que $98 \%$ das crianças estavam frequentando a escola, fato que pode ser explicado pela obrigatoriedade da frequência escolar das crianças para o recebimento do bolsa família, uma vez que este benefício é a segunda principal fonte de renda destas comunidades.

Dentre os atores envolvidos na cadeia produtiva do açaí, percebe-se o alto número dos comunitários que comercializam os frutos in natura. Isso se deve pelo fato da comunidade Murumuru estar um pouco mais distante do principal centro consumidor de açaí na região (área urbana da cidade de Santarém) e as condições ruins das vias para escoamento da produção, o que dificulta o transporte do produto beneficiado. Além disso, alguns comunitários relataram que não possuem condições financeiras para adquirir a máquina despolpadeira de açaí.

Os atravessadores, por sua vez, têm uma participação significativa na cadeia produtiva do açaí em Murumuru porque geralmente nas comunidades quilombolas da região, seus produtos são vendidos por valores muito baixos a atravessadores, que os comercializam em Santarém. Silva et al. (2008) relatam que este problema é determinado pelo isolamento da comunidade e pela precariedade dos meios de transporte público. Além disso, destacam que na estação chuvosa, o escoamento da produção é dificultado pelas precárias condições das vias, que são parcialmente solucionadas pelo uso de transporte fluvial, dependente das condições meteorológicas.

Em relação à renda por ator da cadeia produtiva do açaí, nota-se que os atores que comercializam os frutos in natura (incluindo os atravessadores) obtêm renda maior com o açaí do que aqueles que comercializam só a polpa. Em virtude disso, a atividade 'taberna' tem uma elevada representatividade para os atores que comercializam a polpa, pois estes comercializam a polpa na própria comunidade neste tipo de estabelecimento. Além disso, os atravessadores têm que complementar sua renda com empregos porque são os que mais sofrem no período da entressafra do açaí com a queda da disponibilidade do produto.

\section{CONCLUSÕES}

Apesar da pesca ser a atividade remunerada principal desempenhada pelo maior número de quilombolas em Murumuru, o açaí é a que mais contribui na renda familiar mensal, evidenciando a importância do potencial da comunidade para o extrativismo desse produto. Os atores identificados na cadeia produtiva do açaí na comunidade Murumuru foram: coletor e comerciante dos frutos in natura, atravessador, coletor e comerciante dos frutos in natura e da polpa e coletor e comerciante da polpa. Os quilombolas na comunidade Murumuru que comercializam os frutos de açaí in natura possuem maior renda com esta atividade do que aqueles que comercializam a polpa de açaí. 


\section{REFERÊNCIAS}

ARAÚJO, A. S.; ANJOS, D. R.; SILVA, R. S.; SANTOS, M. A. S.; MARTINS, C. M.; ALMEIDA, R. H. C.. Análise socioeconômica de agricultores da comunidade quilombola do Abacatal, Ananindeua, estado do Pará, Brasil. Biota Amazônia, v.7, n.1, p.30-37, 2017. DOI: http://doi.org.10.18561/21795746/biotaamazonia.v7n1p30-37

BIERNACKI, P.; WALDORF, D.. Snowball Sampling: Problems and techniques of Chain Referral Sampling. Sociological Methods \& Research, v.10, n.2, p.141-163, 1981. DOI: http://doi.org/10.1177/004912418101000205

BRONDÍZıO, E. S.. De alimentação básica para alimentação de moda: ciclos e oportunidades de mudanças no desenvolvimento da economia do açaí no estuário Amazônico. In: ZARIN, D. J.. As florestas produtivas nos neotrópicos. Conservação por meio do manejo sustentável? São Paulo: Peirópolis; Brasília: IEB, 2005. p.427-57.

CARRIL, L. F. B.. Quilombo, Território e Geografia. Agrária, São Paulo, v.3, n.3, p.156-171, 2006.

CONCEIÇÃO, S. S.; SOUZA, B. D.; SANTOS, Y. A.; CONCEIÇÃO, S. S.; RODRIGUES, T. C. A.. O quilombo de São José de Icatu: práticas agrícolas e estratégias de reprodução e manutenção familiar no campo. Enciclopédia Biosfera, v.10, p.19-1367, 2015.

DIAS, B. F. S.. Balanço da biodiversidade na Amazônia: uma introdução ao desconhecido. Seminário Especial: 'A Biodiversidade como Estratégia Moderna de Desenvolvimento da Amazônia'. Rio de Janeiro: INAE, 2001.

DIEGUES, A. C.; VIANA, M. V.. Comunidades tradicionais e manejo dos Recursos naturais da Mata Atlântica. 2 ed. São Paulo: NUPAUB-USP, 2004.

EMBRAPA. Empresa Brasileira de Pesquisa Agropecuária. Serviço Nacional de Levantamento e Conservação de Solos, Rio de Janeiro, RJ. Caracterização dos solos da área do planalto de Se/terra, município de Santarém, Estado do Pará. Belém: Embrapa Amazônia Oriental, 2001.

IBGE. Instituto Brasileiro de Geografia e Estatística. Produção da Extração Vegetal e da Silvicultura. 2017.

PEDROSO JÚNIOR, N. N. P.; MURRIETA, R. S. S.; TAQUEDA, C. S.; NAVAZINAS, N. D.; RUIVO, A. P.; BERNARDO, D. V.; NEVES, W. A.. A casa e a roça: socioeconomia, demografia e agricultura em populações quilombolas do Vale do Ribeira, São Paulo, Brasil. Boletim do Museu Paraense Emílio Goeldi, v.3, n.2, p.227-252, 2008.
LESCURE, J. P.. Algumas questões a respeito do extrativismo. In: EMPERAIRE, L.. A floresta em jogo: o extrativismo na Amazônia Central. São Paulo: UNESP, 2000.

NYGREN, A.; LACUNA-RICHMAN, C.; KEINANEN, K.; ALSA, L.. Ecological, socio-cultural, economic and political factors influencing the contribution of non-timber forest products to local livelihoods: case studies from Honduras and the Philippines. Smallscale Forest Economics, Management and Policy, v.5, n.2, p.249-269, 2006.

REGO, J. F.. Amazônia: do extrativismo ao neoextrativismo. Ciência Hoje, v.25, n.147, p.62-65, 1999.

ROCHA, E.. Potencial ecológico para o manejo de frutos de açaizeiro (Euterpe precatoria Mart.) em áreas extrativistas no Acre, Brasil. Acta Amazônica, v.34, n.2, p.237-250, 2004.

SILVA, D. O.; GUERRERO, A. F. H.; GUERRERO, C. H.; ROLEDO, L. M.. A rede de causalidade da insegurança alimentar $\mathrm{e}$ nutricional de comunidades quilombolas com a construção da rodovia BR-163, Pará, Brasil. Revista de Nutrição, v.21, p.83-97, 2008.

SILVA, T. V. A.; TAPAJÓ, M. P. F.; FURTADO, L. H. S.; ESCHER, S. K. S.; BOGER, A. E.. Estudo etnobotânico em comunidades quilombolas do oeste do Pará. In: CONGRESSO DE CIÊNCIA E TECNOLOGIA DA AMAZÔNIA, 7; SALÃO DE PESQUISA E INICIAÇÃO CIENTÍFICA, 9. Anais. Santarém: CEULS/ULBRA, 2011. p.124-128.

TAKANASHI, S. Y. L.. Níveis de exposição ao mercúrio e desenvolvimento neuropsicomotor em crianças de comunidades quilombolas da região do Baixo Amazonas. Tese (Doutorado em Doenças Tropicais) - Universidade Federal do Pará, Belém, 2014.

VAZ, E. M.; ZACARDI, D. M.; RABELO, Y. G. S.; CORRÊA, J. M. S.. A pesca artesanal no lago Maicá: aspectos socioeconômicos e estrutura operacional. Biota Amazônia, Macapá, v.7, n.4, p.6-12, 2017.

WHA. World Health Association. Division of Mental Health. Qualitative Research for Health Programmes. Geneva: WHA, 1994.

YUYAMA, L. K. O.; AGUIAR, J. P. L.; SILVA FILHO, D. F.; YUYAMA, K.; VAREJÃO, M. J.; FÁVARO, D. I. T.; VASCONCELLOS, M. B. A.; PIMENTEL, S. A.; CARUSO, M. S. F.. Caracterização físico-química do suco de açaí de Euterpe precatoria Mart. oriundo de diferentes ecossistemas amazônicos. Acta Amazônica, v.41, n.4, p.545-552, 2011.

A CBPC - Companhia Brasileira de Produção Científica (CNPJ: 11.221.422/0001-03) detém os direitos materiais desta publicação. Os direitos referem-se à publicação do trabalho em qualquer parte do mundo, incluindo os direitos às renovações, expansões e disseminações da contribuição, bem como outros direitos subsidiários. Todos os trabalhos publicados eletronicamente poderão posteriormente ser publicados em coletâneas impressas sob coordenação da Sustenere Publishing, da Companhia Brasileira de Produção Científica e seus parceiros autorizados. Os (as) autores (as) preservam os direitos autorais, mas não têm permissão para a publicação da contribuição em outro meio, impresso ou digital, em português ou em tradução. 